\title{
Sensing and Detection of Traffic Status through V2V Routing Hop Count and Route Energy
}

\author{
Mahmoud Zaki Iskandarani \\ Faculty of Engineering \\ Al-Ahliyya Amman University, Amman, Jordan
}

\begin{abstract}
New approach to manage congestion using vehicular communication is presented in this work. The research work using MATLAB simulation, tracked communicating vehicles travelling on roads with constant registration of changes in routes, number of hops, and energy consumed as a function of travelled distances. The area of travel and simulation is divided into blocks or zones to enable sufficient allocation and distribution of Road Side Units (RSUs) that are used to relay communication signals and transmission of Basic Safety Messages (BSMs). The successfully concluded simulation is based on the assumption that as congestion occurs, the number of hops per route and associated energy consumption per transmitted packets will change patterns in terms of hops, routes and consumed energy as traffic passes from low to smooth (optimal) to high density (congestion) states, where at the start of congestion, vehicles start to slow down and become closer to each other in a two dimensional space. The output is used as input to traffic status pattern characterization algorithm (management system) that uses the data to indicate the start of traffic accumulation, thus pre-emptive measures can be taken to avoid congestion and reduction in mobility. The presented analysis proved that it is possible to predict congestion as a function of both hops sequences and consumed energy, depending on the hops pattern which is shown to be symmetric in the case of optimum traffic that flows smoothly. The analysis also showed that when congestion starts to occur, asymmetric hops pattern occurs with hops sequences elements switch and swap places within the identified pattern. Further analysis and polynomial curve fitting proved that congestion control and smooth traffic management using the proposed approach is achievable.
\end{abstract}

Keywords-V2V; consumed energy; congestion; hops; VANET; routing

\section{INTRODUCTION}

Mobile data is a critical issue in today's dynamic, fast moving environment. Data transmission requires a network of devices or nodes and links to enable information delivery. One of the most important areas of mobile data application is traffic management and interaction between infrastructure and travelling vehicles. Intelligent transportation system (ITS) enables a more optimized performance of transportation systems through the integration of advanced communication and sensor technologies [1], [2], [3].

A main component of intelligent transportation system is Vehicular Ad Hoc Network (VANET). VANETs are based on similar principles to mobile ad hoc networks (MANETs), where instantaneous formation of a wireless network is carried out in order to enable vehicular data exchange and delivery within a smart vehicular-infrastructure domain. VANETs are enabled through the use of on-board-units (OBUs) installed in the vehicles and road-side-units (RSUs) installed along side roads [4], [5], [6], [7], [8].

Intelligent transportation systems for vehicular ad-hoc networks motivated a wide range of protective applications, such as vehicle collision warning, lane changing, driver assistance, automatic parking, among others. All of the mentioned applications aim to provide, safety, mobility, efficiency, and comfortable transportation, with main objective of reducing accidents and congestion on the roads that result in many cases from unsafe driving, weather conditions, traffic incidents, or simply from traffic mismanagement. Not to forget drivers conditions, attitudes and experiences [9], [10], [11].

The Road traffic faces congested situations that can lead to chaos. Use of connected vehicles that share information and work cooperatively, would result in safer and greener transportation system. Communication systems used to connect vehicles under cooperative driving principles with intelligent controlling algorithms, plays a critical role in traffic monitoring and management. Such technologies assist in the way that infrastructure is designed and built for a smarter environment. The developed wireless communications standards are now in a state where it can be effectively utilized to enable vehicular communication through Vehicular Ad-Hoc Networking [12], [13].

The main proposed wireless communication technology for vehicular connectivity is the Dedicated Short Range Communication (DSRC), which is mapped through the IEEE 802.11p. Such technology enables both Vehicle-to-Vehicle (V2V) and Vehicle-to-Infrastructure (V2I) communication [14], [15], [16], [17]. VANET communication is achieved using dedicated communications devices as part of the OnBoard Units (OBUs) in the vehicles, enabling ad hoc networking among vehicles and between vehicles and infrastructure. This will give the ability to monitor vehicles movements and traffic patterns in order to manage safer, less congested and more reliable traffic motion. Effective VANET communications comprises the use of fast and reliable transmission of Basic safety Messages (BSM) to other vehicles on the road. The BSM should be communicated to sufficient number of vehicles in the shortest possible time with considerations to both consumed energy and bandwidth parameters [18], [19], [20], [21].

Wireless ad hoc networks require routing and management protocols, aims at reducing energy consumption together with 
self-configuration. They apply real time messaging between vehicles (nodes) using radio wave transmission over specific distance (range). VANETS consist of vehicular mobile nodes, established without a centralized infrastructure, thus, each node will perform the functions of transmitter, receiver, and data router [22], [23], [24], [25], [26], [27], [28].

\section{RELATED WORK}

Congestion control is a serious issue when dealing with traffic management. Ideally, vehicles would send BSM to other vehicles within a certain range in order to perform certain tasks required by the employed safety application. However, VANETs encounter several challenges due to various obstacles such as transmission delay, available bandwidth, multi-path fading, among others, which affect the performance of a formed network and associated applications [29], [30], [31].

The use of connected and automated vehicles will impose more pressure on reliability, latency, bandwidth conditions, and threshold levels in terms of vehicular communication. Such requirements will be even more stringent when autonomous vehicles are used, especially when entering or leaving roads under different weather and road conditions, during accidents, when changing lanes, among other conditions and under different scenarios [32], [33], [34].

When designing or modifying the management strategies of transportation network, it is essential to evaluate current performance and multiple scenarios, in order to optimize its operations and account for changing capacity in terms of vehicles and also in relation to wireless communication requirements, such as bandwidth, energy, time, and latency. This can be achieved through simulations using various routing algorithms in order to apply optimization to exchanged control message (BSMs) with minimal time delay, and number of hops among others [35], [36].

In this paper, an approach is proposed which uses transmitted bit energy, elapsed signal routing time, and number of hops, to enable better traffic management and congestion control in response to traffic density, which is a function of the increase in the number of vehicles per travelled area over time intervals. The aim here is to use such parameters as input variables to predict and alleviate road congestion, through route change, traffic lights timing change to better manage the existing traffic conditions in relation to infrastructure.

The rest of this paper is divided as follows: Methodology, Results, Analysis and Discussion, Conclusions, References.

\section{METHODOLOGY}

The main idea of this work is based on the hypothesis that when traffic is very low and traffic density is below a certain threshold with high vehicle mobility (speed, maneuver, lane changing), $\mathrm{V} 2 \mathrm{~V}$ communication will need to use longer routes with fewer connections between travelling vehicles. This means that the number of hops will increase in response to the reduction in available connections and available routes due to fewer available vehicles (nodes) with abundance of space between the travelling vehicles. However, as the traffic density grows and number of travelled vehicles increase per travelled distance, it is expected that the number of connections and available communication routes will increase, which result in a decrease in the number of hops per route, as there will be more available connections due to the presence of more vehicles or nodes and less unoccupied spaces on the road. However, when the traffic is optimal and flowing smoothly and efficiently with maximum utilization of the road, then the number of hops and travelled routes should display a unique balanced characteristic that can be used to detect congestion. These assumptions are to be used in this work as indicators to predict congestion and enable better traffic flow and management. This is based on using the following parameters to characterize traffic condition on the roads:

1) Number of Vehicles (Nodes): $\mathrm{N}$

2) Hops Count: $\mathrm{H}$

3) Message Travelled Distance (Route): MTD

4) Connections Sequence: CS

5) Energy Consumed per Route: ECR

Fig. 1 presents the proposed management system. The system uses the mentioned parameters as inputs to decide if there is a congestion, and act upon such characterization. The simulation traces BSM wireless communication between two specific vehicles (nodes) and analyze the effect of congestion on their connectivity.

Using MATLAB code, the proposed system correlates the number of travelling vehicles $(\mathrm{N})$ to:

1) Hops Count $(\mathrm{H})$.

2) Message Travelled Distance (MTD).

3) Energy Consumed per Route (ECR).

4) Connections Sequences (CS).

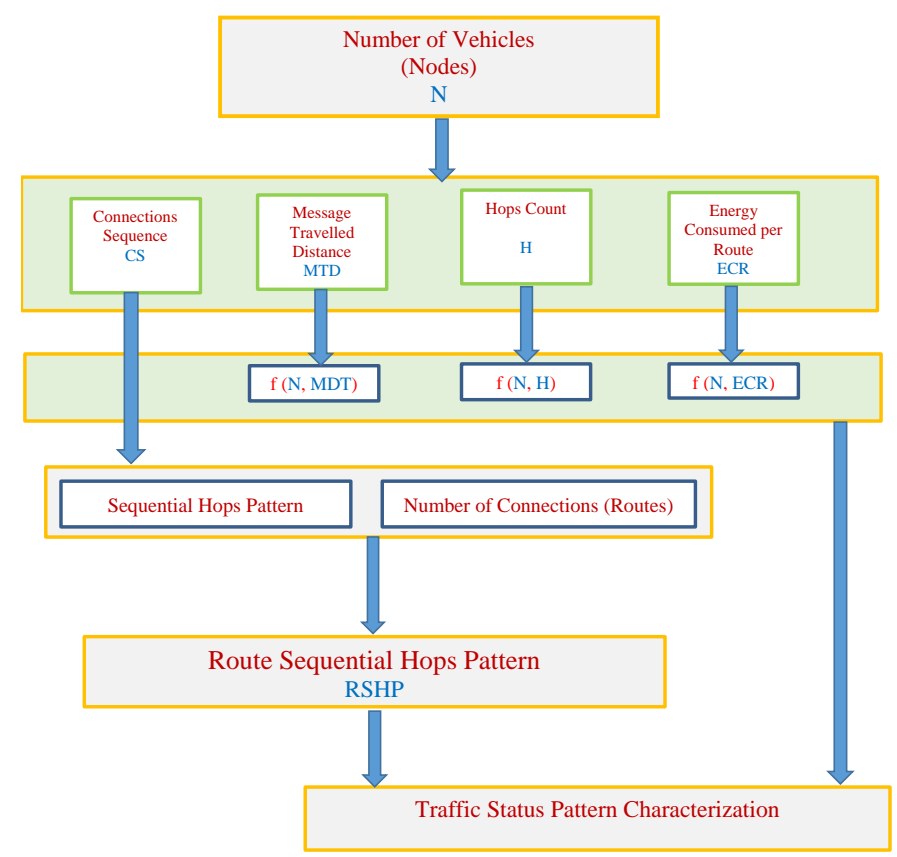

Fig. 1. Proposed Management System. 
Connection Sequences are further used to establish connectivity pattern that is implicitly related to Hops Count (H), Message Travelled Distance (MTD), and the Energy Consumed per Route (ECR) through monitoring of repetitive number of hops for each single connection and over the whole travelled route, which is projected onto two parameters:

1) Sequential Hops Patterns

2) Number of Connections (Routes)

Both Sequential Hops Patterns and Number of Connection (Routes) are then used per number of vehicles in a scenario that is simulated in urban area to produce the Route Sequential Hops Pattern (RSHP) for the state of traffic, enabling traffic density and congestion level characterization through correlation with MTD, $\mathrm{H}$, and ECR all as a function of number of travelling vehicles. This will eliminate errors in traffic characterization and management, when the driver is driving slowly by choice and not as a result of congestion.

The proposed approach eliminates the need to consider travelling time as roads speed differ in different areas and is a function of road design and purpose. Thus making the presented model and simulation applicable to any road type and structure.

As vehicular communication covers both $\mathrm{V} 2 \mathrm{~V}$ and V2I, with $\mathrm{V} 2 \mathrm{~V}$ is also accomplished as a result of V2I communication to communicate certain data obtained from Road side Units (RSUs), then it is necessary to establish communication zones over the physical roads to enable determination of transmission ranges within each zone or area of vehicular travel, so that coverage is guaranteed and signal strength is within acceptable limits.

The travelled area or area size is divided into zones of 40 $\mathrm{m}$ by $40 \mathrm{~m}$. The proposed and simulated block division allows for addition and operating of RSUs as a function of the total travel distance by vehicles.

The transmission range in one dimension (Longitudinally or laterally) is specified according to equation (1):

\section{Transmission Range $(T R)=\beta * Z S$}

Where $\beta \leq 1$ and $Z S:$ Zone Size

The condition of $\beta$ to be less than one is essential to guarantee non-interference of communicating vehicles groups within each zone and to enable more accurate traffic management especially in the cases of work zones and accidents zones. However, as vehicles are moving there will be no interruption of communications as they move from one zone to the next, very similar to cellular communication that uses cells and handover regions from one coverage to the other. The ratio can be one only if the area is considered as one zone covered by one RSU.

From equation (1), Transmission Range (TR) can be related to Message Travelled Distance (MTD) through equation (2).

Transmission Range $(T R) \geq M T D$
Thus the dynamic MTD for a direct V2V communication between any two vehicles can be approximated as in equation (3).

$$
M T D \leq(\beta * Z S)
$$

From equation (3), the consumed energy is approximated by equation (4).

$$
E C R_{\text {consumed }} \leq\left(E C R^{*} \beta * Z S\right)
$$

Each Hop will consume energy within the specified zone according to equation (5).

$$
E C R_{\text {consumed (Hop ) }} \leq\left(\frac{E C R^{*} \beta^{*} Z S}{H}\right)
$$

In this work the used ratio is 0.75 as more than one zone is considered in the simulation as shown in Fig. 2. The transmission range is dynamic and a function of vehicles movements and time. Hence, it is of great advantage to subdivide the travelled area into Zones in case of temporary vehicular groups, where it becomes much easier to manage their communications as they move and leave one group to enter another temporary group that belongs to a different zone.

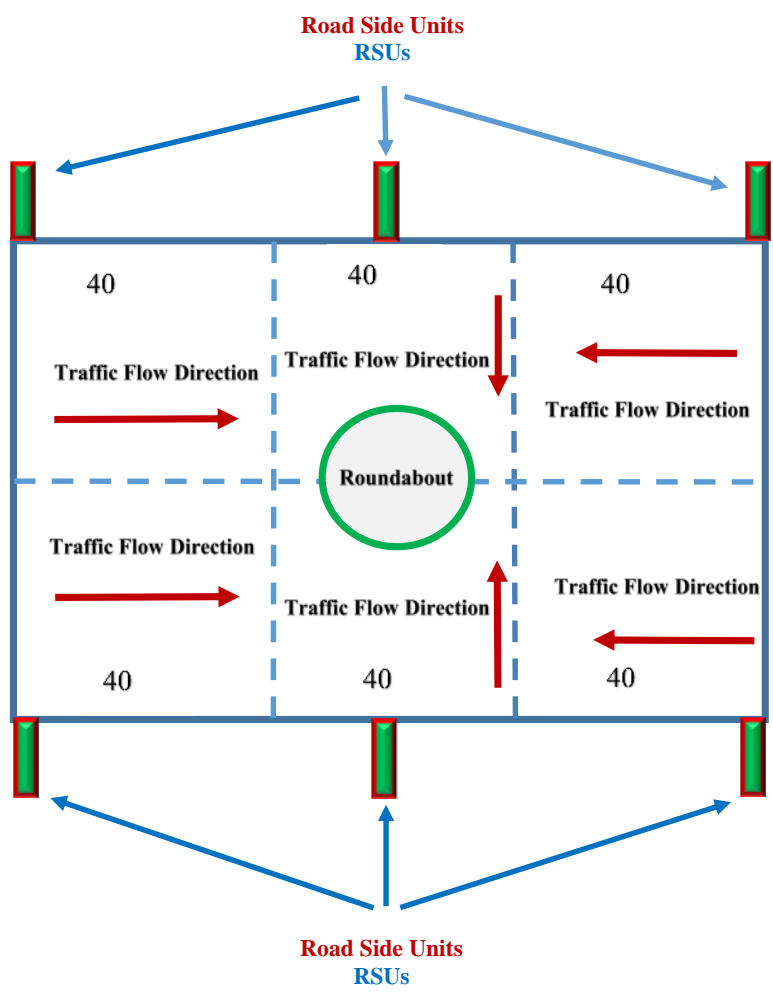

Fig. 2. Simulated Area with Divided Blocks (Zones).

\section{RESULTS}

Tables I to III present the initial simulation results with traffic density or vehicles numbers (nodes): $\{40,280,440\}$. 
TABLE I. ENERGY VARIATION AS A FUNCTION OF NUMBER OF

CONNECTIONS FOR 40 VEHICLES

\begin{tabular}{|c|c|c|c|}
\hline $\begin{array}{l}\text { Connections } \\
\text { Sequence }\end{array}$ & Hops Count & $\begin{array}{l}\text { Message Travelled } \\
\text { Distance }(\mathrm{m})\end{array}$ & $\begin{array}{l}\text { Consumed Energy } \\
\text { Per Route }(J)\end{array}$ \\
\hline 1 & 3 & 72.83 & 0.0015 \\
\hline 2 & 3 & 53.91 & 0.0009 \\
\hline 3 & 3 & 52.74 & 0.0008 \\
\hline 4 & 3 & 52.29 & 0.0008 \\
\hline 5 & 3 & 52.40 & 0.0008 \\
\hline 6 & 3 & 52.82 & 0.0008 \\
\hline 7 & 3 & 53.43 & 0.0009 \\
\hline 8 & 3 & 54.17 & 0.0009 \\
\hline 9 & 3 & 55.01 & 0.0009 \\
\hline 10 & 3 & 55.95 & 0.0009 \\
\hline 11 & 3 & 56.99 & 0.0010 \\
\hline 12 & 3 & 58.11 & 0.0010 \\
\hline 13 & 3 & 59.32 & 0.0010 \\
\hline 14 & 3 & 60.63 & 0.0011 \\
\hline 15 & 3 & 62.02 & 0.0011 \\
\hline 16 & 3 & 63.50 & 0.0012 \\
\hline 17 & 3 & 65.07 & 0.0012 \\
\hline 18 & 3 & 66.73 & 0.0013 \\
\hline 19 & 3 & 68.46 & 0.0013 \\
\hline 20 & 3 & 70.28 & 0.0014 \\
\hline 21 & 3 & 72.16 & 0.0015 \\
\hline 22 & 3 & 74.12 & 0.0015 \\
\hline 23 & 3 & 76.15 & 0.0016 \\
\hline 24 & 3 & 78.23 & 0.0017 \\
\hline 25 & 4 & 94.34 & 0.0024 \\
\hline 26 & 4 & 95.11 & 0.0024 \\
\hline 27 & 4 & 96.11 & 0.0025 \\
\hline 28 & 4 & 97.31 & 0.0026 \\
\hline 29 & 4 & 98.70 & 0.0026 \\
\hline 30 & 4 & 90.95 & 0.0022 \\
\hline 31 & 4 & 101.57 & 0.0028 \\
\hline 32 & 4 & 103.15 & 0.0029 \\
\hline 33 & 4 & 104.88 & 0.0029 \\
\hline 34 & 4 & 93.74 & 0.0024 \\
\hline
\end{tabular}

TABLE II. ENERGY VARIATION AS A FUNCTION OF NUMBER OF CONNECTIONS FOR 280 VEHICLES

\begin{tabular}{|l|l|l|l|}
\hline $\begin{array}{l}\text { Connections } \\
\text { Sequence }\end{array}$ & $\begin{array}{l}\text { Hops } \\
\text { Count }\end{array}$ & $\begin{array}{l}\text { Message Travelled } \\
\text { Distance }(\mathrm{m})\end{array}$ & $\begin{array}{l}\text { Consumed Energy Per } \\
\text { Route }(\mathrm{J})\end{array}$ \\
\hline 1 & 4 & 110.40 & 0.0032 \\
\hline 2 & 4 & 107.98 & 0.0031 \\
\hline 3 & 4 & 105.59 & 0.0030 \\
\hline 4 & 4 & 103.25 & 0.0029 \\
\hline 5 & 4 & 100.97 & 0.0027 \\
\hline 6 & 4 & 98.91 & 0.0026 \\
\hline 7 & 4 & 97.03 & 0.0025 \\
\hline 8 & 4 & 95.17 & 0.0024 \\
\hline 9 & 4 & 93.34 & 0.0024 \\
\hline 10 & 4 & 94.61 & 0.0024 \\
\hline 11 & 4 & 99.45 & 0.0027 \\
\hline 12 & 4 & 98.12 & 0.0026 \\
\hline 13 & 3 & 87.73 & 0.0021 \\
\hline 14 & 3 & 82.18 & 0.0019 \\
\hline
\end{tabular}

\begin{tabular}{|c|c|c|c|}
\hline 15 & 3 & 80.76 & 0.0018 \\
\hline 16 & 3 & 79.47 & 0.0017 \\
\hline 17 & 3 & 78.31 & 0.0017 \\
\hline 18 & 3 & 75.54 & 0.0016 \\
\hline 19 & 3 & 80.36 & 0.0018 \\
\hline 20 & 3 & 72.04 & 0.0015 \\
\hline 21 & 3 & 70.38 & 0.0014 \\
\hline 22 & 3 & 69.41 & 0.0014 \\
\hline 23 & 3 & 68.06 & 0.0013 \\
\hline 24 & 3 & 66.86 & 0.0013 \\
\hline 25 & 3 & 65.87 & 0.0012 \\
\hline 26 & 3 & 71.58 & 0.0014 \\
\hline 27 & 3 & 71.94 & 0.0015 \\
\hline 28 & 3 & 76.52 & 0.0016 \\
\hline 29 & 3 & 76.47 & 0.0016 \\
\hline 30 & 2 & 55.25 & 0.0009 \\
\hline 31 & 2 & 53.84 & 0.0009 \\
\hline 32 & 2 & 52.50 & 0.0008 \\
\hline 33 & 2 & 51.25 & 0.0008 \\
\hline 34 & 2 & 50.10 & 0.0008 \\
\hline 35 & 2 & 49.07 & 0.0007 \\
\hline 36 & 2 & 48.18 & 0.0007 \\
\hline 37 & 2 & 47.44 & 0.0007 \\
\hline 38 & 2 & 46.86 & 0.0007 \\
\hline 39 & 2 & 46.47 & 0.0007 \\
\hline 40 & 2 & 46.27 & 0.0007 \\
\hline 41 & 2 & 46.27 & 0.0007 \\
\hline 42 & 2 & 45.69 & 0.0007 \\
\hline 43 & 2 & 45.48 & 0.0007 \\
\hline 44 & 2 & 45.31 & 0.0007 \\
\hline 45 & 2 & 45.16 & 0.0007 \\
\hline 46 & 2 & 45.04 & 0.0006 \\
\hline 47 & 2 & 44.94 & 0.0006 \\
\hline 48 & 2 & 44.86 & 0.0006 \\
\hline 49 & 2 & 44.80 & 0.0006 \\
\hline 50 & 2 & 44.76 & 0.0006 \\
\hline 51 & 2 & 44.73 & 0.0006 \\
\hline 52 & 2 & 44.72 & 0.0006 \\
\hline 53 & 2 & 49.27 & 0.0007 \\
\hline 54 & 2 & 50.64 & 0.0008 \\
\hline 55 & 2 & 52.09 & 0.0008 \\
\hline 56 & 2 & 63.41 & 0.0012 \\
\hline 57 & 2 & 63.42 & 0.0012 \\
\hline 58 & 3 & 60.26 & 0.0011 \\
\hline 59 & 3 & 67.24 & 0.0013 \\
\hline 60 & 3 & 69.24 & 0.0014 \\
\hline 61 & 3 & 81.21 & 0.0018 \\
\hline 62 & 3 & 73.24 & 0.0015 \\
\hline 63 & 4 & 76.40 & 0.0016 \\
\hline 64 & 4 & 77.70 & 0.0017 \\
\hline 65 & 4 & 96.36 & 0.0025 \\
\hline 66 & 4 & 96.44 & 0.0025 \\
\hline 67 & 4 & 96.61 & 0.0025 \\
\hline 68 & 4 & 96.86 & 0.0025 \\
\hline 69 & 4 & 97.19 & 0.0025 \\
\hline 70 & 4 & 97.61 & 0.0026 \\
\hline 71 & 4 & 98.13 & 0.0026 \\
\hline
\end{tabular}


TABLE III. ENERGY VARIATION AS A FUNCTION OF NUMBER OF CONNECTIONS FOR 440 VEHICLES

\begin{tabular}{|c|c|c|c|}
\hline $\begin{array}{l}\text { Connections } \\
\text { Sequence }\end{array}$ & Hops Count & $\begin{array}{l}\text { Message Travelled } \\
\text { Distance }(\mathrm{m})\end{array}$ & $\begin{array}{l}\text { Consumed Energy } \\
\text { Per Route }(\mathrm{J})\end{array}$ \\
\hline 1 & 3 & 67.32 & 0.0013 \\
\hline 2 & 3 & 65.98 & 0.0012 \\
\hline 3 & 3 & 64.73 & 0.0012 \\
\hline 4 & 2 & 63.59 & 0.0012 \\
\hline 5 & 2 & 58.77 & 0.0010 \\
\hline 6 & 2 & 57.32 & 0.0010 \\
\hline 7 & 2 & 55.87 & 0.0009 \\
\hline 8 & 2 & 54.49 & 0.0009 \\
\hline 9 & 2 & 53.20 & 0.0009 \\
\hline 10 & 2 & 52.00 & 0.0008 \\
\hline 11 & 2 & 50.91 & 0.0008 \\
\hline 12 & 2 & 49.95 & 0.0008 \\
\hline 13 & 2 & 49.13 & 0.0007 \\
\hline 14 & 2 & 48.47 & 0.0007 \\
\hline 15 & 2 & 47.98 & 0.0007 \\
\hline 16 & 2 & 47.69 & 0.0007 \\
\hline 17 & 2 & 47.59 & 0.0007 \\
\hline 18 & 2 & 47.69 & 0.0007 \\
\hline 19 & 2 & 47.98 & 0.0007 \\
\hline 20 & 2 & 48.47 & 0.0007 \\
\hline 21 & 2 & 49.13 & 0.0007 \\
\hline 22 & 2 & 49.95 & 0.0008 \\
\hline 23 & 2 & 50.91 & 0.0008 \\
\hline 24 & 2 & 52.00 & 0.0008 \\
\hline 25 & 2 & 53.20 & 0.0009 \\
\hline 26 & 2 & 54.49 & 0.0009 \\
\hline 27 & 2 & 55.87 & 0.0009 \\
\hline 28 & 2 & 57.32 & 0.0010 \\
\hline 29 & 2 & 45.39 & 0.0007 \\
\hline 30 & 2 & 40.86 & 0.0006 \\
\hline 31 & 2 & 41.28 & 0.0006 \\
\hline 32 & 2 & 41.78 & 0.0006 \\
\hline 33 & 2 & 42.34 & 0.0006 \\
\hline 34 & 2 & 42.96 & 0.0006 \\
\hline 35 & 2 & 43.66 & 0.0006 \\
\hline 36 & 2 & 44.42 & 0.0006 \\
\hline 37 & 2 & 45.26 & 0.0007 \\
\hline 38 & 2 & 46.18 & 0.0007 \\
\hline 39 & 2 & 47.18 & 0.0007 \\
\hline 40 & 2 & 48.26 & 0.0007 \\
\hline 41 & 2 & 49.43 & 0.0008 \\
\hline 42 & 2 & 50.69 & 0.0008 \\
\hline 43 & 3 & 52.04 & 0.0008 \\
\hline 44 & 3 & 55.22 & 0.0009 \\
\hline 45 & 3 & 56.11 & 0.0009 \\
\hline 46 & 3 & 57.25 & 0.0010 \\
\hline 47 & 3 & 58.59 & 0.0010 \\
\hline 48 & 3 & 60.07 & 0.0011 \\
\hline 49 & 3 & 61.65 & 0.0011 \\
\hline 50 & 3 & 63.31 & 0.0012 \\
\hline 51 & 3 & 65.04 & 0.0012 \\
\hline 52 & 3 & 66.81 & 0.0013 \\
\hline
\end{tabular}

\begin{tabular}{|l|l|l|l|}
\hline 53 & 3 & 68.61 & 0.0013 \\
\hline 54 & 3 & 70.44 & 0.0014 \\
\hline 55 & 3 & 72.30 & 0.0015 \\
\hline 56 & 3 & 74.18 & 0.0015 \\
\hline 57 & 3 & 75.32 & 0.0016 \\
\hline 58 & 3 & 76.03 & 0.0016 \\
\hline 59 & 3 & 77.91 & 0.0017 \\
\hline 60 & 3 & 78.08 & 0.0017 \\
\hline 61 & 3 & 79.89 & 0.0018 \\
\hline 62 & 3 & 81.86 & 0.0018 \\
\hline 63 & 3 & 83.99 & 0.0019 \\
\hline 64 & 4 & 86.27 & 0.0020 \\
\hline 65 & 4 & 92.70 & 0.0023 \\
\hline 66 & 4 & 94.70 & 0.0024 \\
\hline 67 & 4 & 96.70 & 0.0025 \\
\hline 68 & 4 & 98.24 & 0.0026 \\
\hline 69 & 4 & 100.24 & 0.0027 \\
\hline 70 & 4 & 102.24 & 0.0028 \\
\hline 71 & 4 & 103.00 & 0.0028 \\
\hline 72 & 4 & 104.32 & 0.0029 \\
\hline & & & \\
\hline
\end{tabular}

\section{ANALYSIS AND DISCUSSION}

Fig. 3 shows the relationship between increasing number of vehicles (nodes) and the average message travelled distance by a transmitted signal from one vehicle to another vehicle. It is clear from the plot that as the traffic density and volume increases per same area size, the travelled distance will decrease, due to vehicles or nodes becoming closer to each other, as their number increases and their speed drops. Hence, the number of 2 hops routes will increase, while 3 hops, and 4 hops routes will decrease, thus reducing the average message travelled distance. This is supported by the detailed hops count and comparative plot shown in Fig. 4.

Fig. 4 present detailed hops distribution that count for each simulated volume of vehicles (nodes) travelling the same areas size. As the plot and associated table present, at 40 vehicles volume, 2 hops routes are not used as the vehicles (nodes) are very far apart (very low traffic), hence only 3 hops and 4 hops connectivity routes are available. As the traffic volume increase to 280 vehicles (nodes), traffic density increases per area size and more vehicles are now able to communicate through 2 hops routes, thus the overall hops count increases in relation to traffic volume with 2 hops routes having larger percentage of the communication routes. However, as the traffic flow is smooth and congestion state is not reached, 3 hops routes and 4 hops routes are almost equally distributed.

With number of vehicles reaching 440 vehicles (nodes) per same area size, the simulated results show a decrease in the 4 hops routes and a large increase in 2 hops routes, with slight increase in 3 hops routes. This is due to the increase in volume and traffic density above normal conditions (optimum level), which lead to vehicles or communicating nodes become closer, slowing down to a limited speed as a result of traffic build up. These observations, which are supported by the carried out MATLAB simulation proves the presented hypothesis and is further backed by the presented plots and associated data in Fig. 5 where, a clear indication of the 
reduction in the average consumed energy as the number of hops per route is reduced going from 40 vehicles (nodes) through a smooth (optimum) traffic density per area size (280 vehicles), and up to the start of congestion, where the number of vehicles reached 440 vehicles or nodes.

Such deduction is further proved in Fig. 6, where the corresponding reduction in the average consumed energy per route is shown to correlate with the increase of the 2 hops routes and the reduction of 3 hops routes and 4 hops routes. All plots and analysis are based on data in Tables I to III.

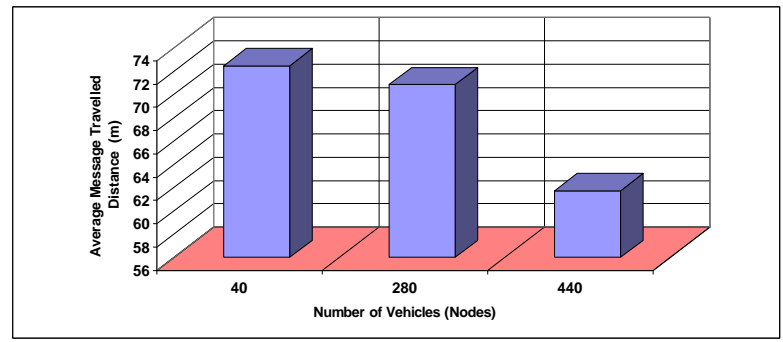

Fig. 3. Effect of Number of Vehicles (nodes) on Average Travelled Distance (Route).

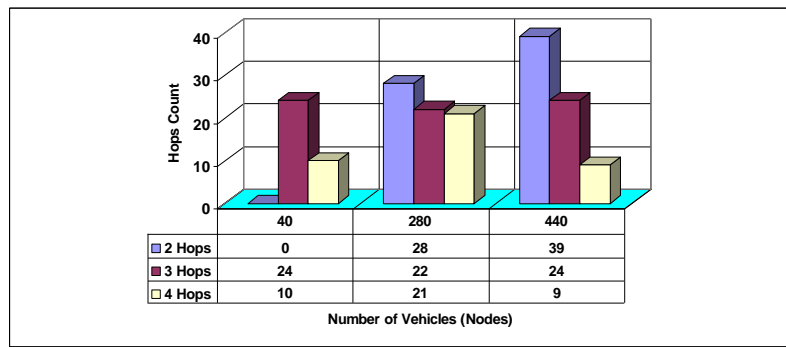

Fig. 4. Relationship between Number of Vehicles (nodes), Hops Distribution and Hop Count.

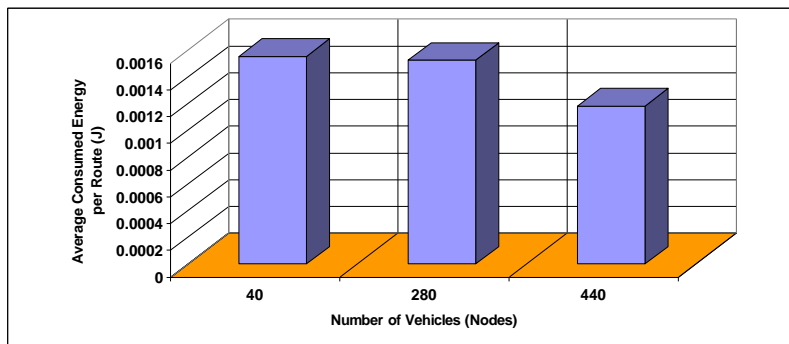

Fig. 5. Effect of Number of Vehicles (nodes) on Average Consumed Energy for Transmitted Bytes.

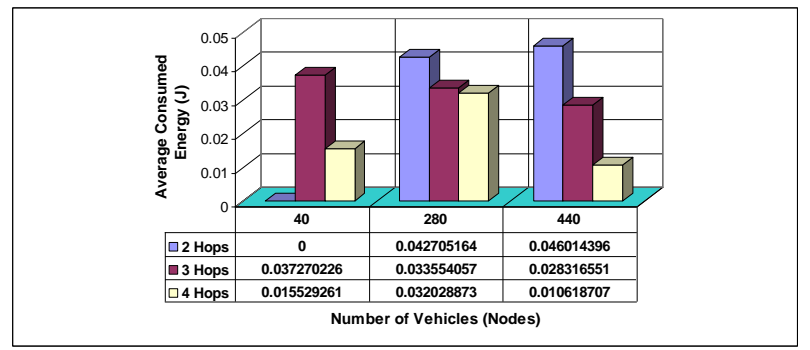

Fig. 6. Relationship between Number of Vehicles (nodes), Hop Distribution and the Average Consumed Energy for Transmitted Bytes.
Based on the previous analysis, the normal pattern for optimum number of vehicles (nodes) in relation to the available area size that results in smooth traffic flow, is related to the sequential hops pattern through symmetrical analysis. A symmetrical transition between routes with different number of hops, indicates smooth traffic with optimum mobility, any deviation from that present the extreme low traffic or very high traffic (congestion). Three cases are depicted by Fig. 7 to 9:

1) If the number of vehicles (nodes) are too low (low traffic flow) in relation to area size: the sequential hops pattern is shown to be [3, 4], (Fig. 7).

2) If the number of vehicles (nodes) are optimum (smooth traffic flow) in relation to area size: the sequential hops pattern is shown to be [4, 3, 2, 3, 4], (Fig. 8).

3 ) If the number of vehicles (nodes) are high (High density traffic flow (congestion) in relation to area size: the sequential hops pattern is shown to be [3, 2, 3, 4, 3], (Fig. 9).

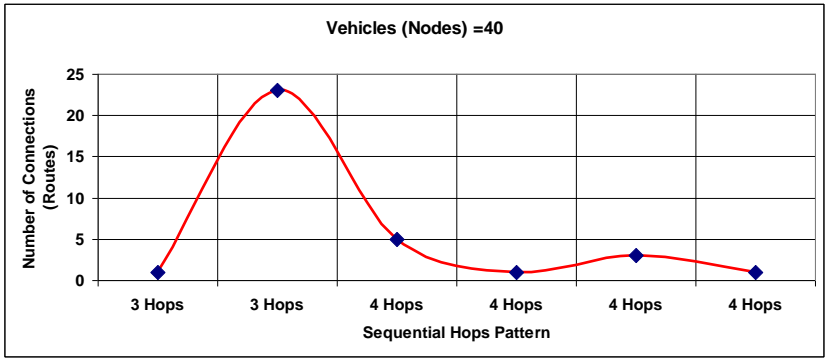

Fig. 7. Hops Distribution Dynamics as a Function of Connections (Routes) for Low Traffic Density.

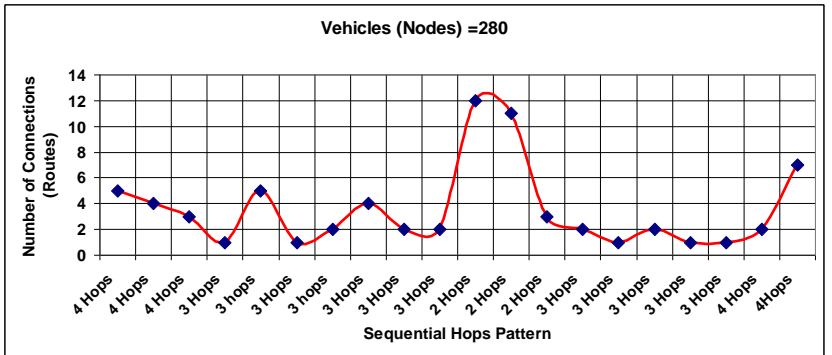

Fig. 8. Hops Distribution Dynamics as a Function of Connections (Routes) for Optimum Traffic Density.

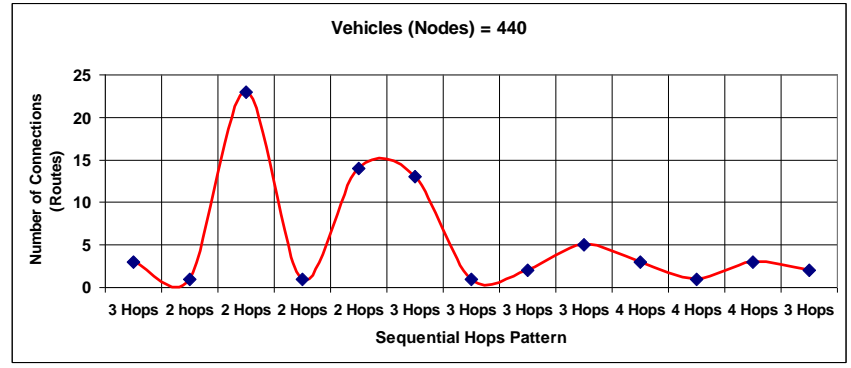

Fig. 9. Hops Distribution Dynamics as a Function of Connections (Routes) for High Traffic Density (Congestion). 
Thus, the symmetric case only occurs when the traffic is optimum (case 1), while when it is very low, the 2 hops connectivity does not exist, as the vehicles are far apart (also taking into account speed). In the case of congestion, a shift in the 2 hops is observed as they swap places and a switch also occurs in places between 4 hops and 3 hops routes (case 3 ). The swapping, switching, and deviation from symmetricity implies a serious change in the traffic flow pattern, which is proved through simulation to indicate congestion. The route sequential hops pattern (RSHP), discussed above can be generalized as in equations (6) to (8), assuming maximum allowed number of hops is $\mathrm{H}_{\max }$.

$$
\begin{aligned}
& R S H P_{\text {low traffic }}=\left[\left(H_{\max }-1\right), \cdots,\left(H_{\max }\right)\right] \\
& R S H P_{\text {smooth traffic }}=\left[\left(H_{\max }\right), \cdots,\left(H_{\max }-1\right),\left(H_{\max }-n\right),\left(H_{\max }-1\right), \cdots,\left(H_{\max }\right)\right] \\
& R S H P_{\text {Congession }}=\left[\left(H_{\max }-1\right),\left(H_{\max }-n\right),\left(H_{\max }-1\right), \cdots,\left(H_{\max }\right), \cdots,\left(H_{\max }-1\right)\right]
\end{aligned}
$$

$\left(H_{\max }-n\right)$ : The minimum number of hops per route, which is assumed to hold a value of 2 .

The expressions in equations (6) to (8) with their associated patterns are correlated to the following variables as indicated in the proposed system in Fig. 1:

\section{1) Hops Count $(\mathrm{H})$}

2) Message Travelled Distance (MTD)

3) Energy Consumed per Route (ECR)

Thus, eliminating any errors that might result when a vehicle is moving at slow speed not because of traffic density, but as a matter of choice.

\section{CONCLUSIONS}

This work presented a new concept in congestion control as a function of hops count and route energy variation. The work attempted to solve a critical issue in traffic management and smart cities, namely, congestion. Congestion is a result of inefficient transportation policies, or a consequence of road incidents, will adversely contribute to the economic, environmental, and societal beings. Hence, it is of prime importance to control congestion and work towards smooth traffic flow. As a result of this work, a final correlation can be made between:

1) The consumed energy per used route and traffic density.

2) The number of connections during vehicle's trip and traffic density.

Fig. 10 and 11 illustrate the relationships with fitted polynomial curves.

The fitted curves mathematical expressions are given in equations (9) and (10).

$$
\begin{aligned}
& M=4 \times 10^{-4} N^{2}+273 \times 10^{-3} N+0.237 \times 10^{2} \\
& E C R=5 \times 10^{-9} N^{2}+2 \times 10^{-6} N+15 \times 10^{-4}
\end{aligned}
$$

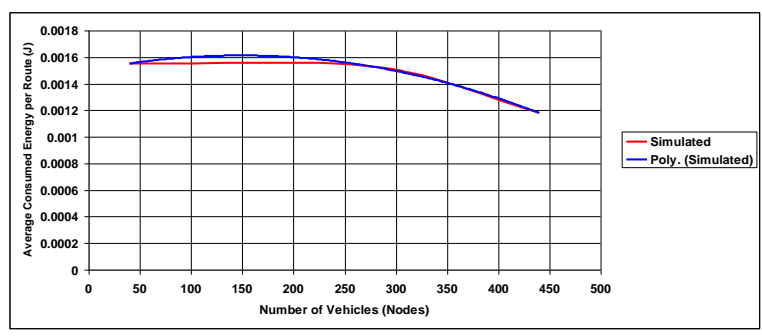

Fig. 10. Effect of Increasing Traffic Density on the Average Consumed Energy Per route.

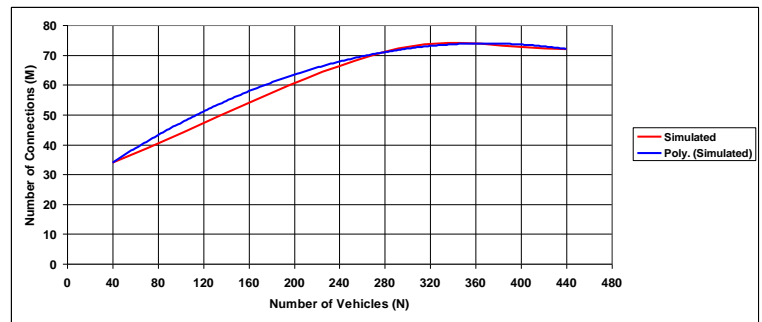

Fig. 11. Effect of Increasing Traffic Density on the Number of Connections Per Trip.

Where;

M: Number of Connections.

ECR: Energy Consumed per Route.

The reduction in energy as nodes number increases is due to slower motion and mobility, which indicates congestion and fewer available connections at the horizon. Also, 2 hops connections are shown to increase as vehicles (nodes) become closer to each other within the available range contributing to lower energy consumption.

Finely, this work achieved the following:

1) Establishing a relationship between congestion and number of hops per communication route as a function of travelling vehicles.

2) Enabling the correlation between physical travelled distance and communication route length.

3) Deducing a relationship between consumed energy and congestion.

4) Proving that energy consumed is a function of number of hops, which is also related to the consumed energy that can be correlated to congestion.

5) Proposing a correlative algorithm that supports traffic management system.

\section{REFERENCES}

[1] S. Gössling, "ICT and transport behavior: A conceptual review," Int. J. Sustain. Transp., vol. 12, no. 3, pp. 153-164, 2018, doi: 10.1080/15568318.2017.1338318.

[2] E. Loos, M. Sourbati, and F. Behrendt, "The role of mobility digital ecosystems for age-friendly urban public transport: A narrative literature review," Int. J. Environ. Res. Public Health, vol. 17, no. 20, pp. 1-16, 2020, doi: 10.3390/ijerph17207465.

[3] Z. Pavlović, M. Banjanin, J. Vukmirović, and D. Vukmirović, "Contactless Ict Transaction Model of the Urban Transport Service," Transport, vol. 0, no. 0, pp. 1-11, 2020, doi: 10.3846/transport.2020.12529. 
[4] F. Arena and G. Pau, "An overview of vehicular communications," Futur. Internet, vol. 11, no. 2, 2019, doi: 10.3390/fi11020027.

[5] M. El Zorkany, A. Yasser, and A. I. Galal, "Vehicle To Vehicle 'V2V' Communication: Scope, Importance, Challenges, Research Directions and Future," Open Transp. J., vol. 14, no. 1, pp. 86-98, 2020, doi: $10.2174 / 1874447802014010086$.

[6] Z. El-Rewini, K. Sadatsharan, D. F. Selvaraj, S. J. Plathottam, and P. Ranganathan, "Cybersecurity challenges in vehicular communications," Veh. Commun., vol. 23, p. 100214, 2020, doi: 10.1016/j.vehcom.2019.100214.

[7] F. Pereira et al., "When Backscatter Communication Meets Vehicular Networks: Boosting Crosswalk Awareness," IEEE Access, vol. 8, pp. 34507-34521, 2020, doi: 10.1109/ACCESS.2020.2974214.

[8] M. S. Sheikh, J. Liang, and W. Wang, "Security and Privacy in Vehicular Ad Hoc Network and Vehicle Cloud Computing: A Survey," Wirel. Commun. Mob. Comput., vol. 2020, 2020, doi: 10.1155/2020/5129620.

[9] Y. Bai, K. Zheng, Z. Wang, X. Wang, and J. Wang, "MC-Safe: Multichannel Real-time V2V Communication for Enhancing Driving Safety," ACM Trans. Cyber-Physical Syst., vol. 4, no. 4, 2020, doi: $10.1145 / 3394961$.

[10] P. Sewalkar and J. Seitz, "Vehicle-to-pedestrian communication for vulnerable road users: Survey, design considerations, and challenges," Sensors (Switzerland), vol. 19, no. 2, 2019, doi: 10.3390/s19020358.

[11] K. Yu, L. Peng, X. Ding, F. Zhang, and M. Chen, "Prediction of instantaneous driving safety in emergency scenarios based on connected vehicle basic safety messages," J. Intell. Connect. Veh., vol. 2, no. 2, pp. 78-90, 2019, doi: 10.1108/jicv-07-2019-0008.

[12] S. A. Ahmad, A. Hajisami, H. Krishnan, F. Ahmed-Zaid, and E. MoradiPari, "V2V System Congestion Control Validation and Performance," IEEE Trans. Veh. Technol., vol. 68, no. 3, pp. 2102-2110, 2019, doi: 10.1109/TVT.2019.2893042.

[13] M. Baek, D. Jeong, D. Choi, and S. Lee, "Vehicle trajectory prediction and collision warning via fusion of multisensors and wireless vehicular communications," Sensors (Switzerland), vol. 20, no. 1, 2020, doi: $10.3390 / \mathrm{s} 20010288$.

[14] Venkatamangarao Nampally and Dr. M. Raghavender Sharma, "A Novel Protocol for Safety Messaging and Secure Communication for VANET System: DSRC," Int. J. Eng. Res., vol. V9, no. 01, pp. 391397, 2020, doi: 10.17577/ijertv9is010029.

[15] Z. Xu, X. Li, X. Zhao, M. H. Zhang, and Z. Wang, "DSRC versus 4GLTE for connected vehicle applications: A study on field experiments of vehicular communication performance," J. Adv. Transp., vol. 2017, 2017, doi: 10.1155/2017/2750452.

[16] Y. A. Vershinin and Y. Zhan, "Vehicle to Vehicle Communication: Dedicated Short Range Communication and Safety Awareness," 2020 Syst. Signals Gener. Process. F. Board Commun., pp. 1-3, 2020, doi: 10.1109/IEEECONF48371.2020.9078660.

[17] S. Kim and B. J. Kim, "Prioritization of Basic Safety Message in DSRC Based on Distance to Danger," arXiv, pp. 1-10, 2020.

[18] B. L. Nguyen, D. T. Ngo, N. H. Tran, M. N. Dao, and H. L. Vu, "Dynamic V2I/V2V Cooperative Scheme for Connectivity and Throughput Enhancement," IEEE Trans. Intell. Transp. Syst., vol. 2020, no. 2, pp. 1-11, 2020, doi: 10.1109/tits.2020.3023708.

[19] H. Kim and T. Kim, "Vehicle-to-vehicle (V2V) message content plausibility check for platoons through low-power beaconing," Sensors (Switzerland), vol. 19, no. 24, pp. 1-20, 2019, doi: 10.3390/s19245493.

[20] J. Liu, W. Yang, J. Zhang, and C. Yang, "Detecting false messages in vehicular ad hoc networks based on a traffic flow model," Int. J. Distrib. Sens. Networks, vol. 16, no. 2, 2020, doi: 10.1177/1550147720906390.
[21] X. Liu and A. Jaekel, "Congestion control in V2V safety communication: Problem, analysis, approaches," Electron., vol. 8, no. 5, 2019, doi: 10.3390/electronics8050540.

[22] C. R. Guerber, E. L. Gomes, M. S. Pereira Fonseca, A. Munaretto, and T. H. Silva, "Transmission Opportunities: A New Approach to Improve Quality in V2V Networks," Wirel. Commun. Mob. Comput., vol. 2019, pp. 1-6, 2019, doi: 10.1155/2019/1708437.

[23] K. Eshteiwi, G. Kaddoum, B. Selim, and F. Gagnon, "Impact of CoChannel Interference and Vehicles as Obstacles on Full-Duplex V2V Cooperative Wireless Network," IEEE Trans. Veh. Technol., vol. 69, no. 7, pp. 7503-7517, 2020, doi: 10.1109/TVT.2020.2993508.

[24] H. Chang, Y. E. Song, H. Kim, and H. Jung, "Distributed transmission power control for communication congestion control and awareness enhancement in VANETs," PLoS One, vol. 13, no. 9, pp. 1-25, 2018, doi: 10.1371/journal.pone.0203261.

[25] M. Z. Iskandarani, "Effect of Route Length and Signal Attenuation on Energy Consumption in V2V Communication," Int. J. Adv. Comput. Sci. Appl., vol. 11, no. 10, pp. 304-309, 2020, doi: 10.14569/ijacsa.2020.0111039.

[26] O. S. Eyobu, J. Joo, and D. S. Han, "A broadcast scheme for vehicle-topedestrian safety message dissemination," Int. J. Distrib. Sens. Networks, vol. 13, no. 11, 2017, doi: 10.1177/1550147717741834.

[27] S. Li, F. Wang, J. Gaber, and X. Chang, "Throughput and Energy Efficiency of Cooperative ARQ Strategies for VANETs Based on Hybrid Vehicle Communication Mode," IEEE Access, vol. 8, pp. 114287-114304, 2020, doi: 10.1109/ACCESS.2020.3003813.

[28] T. Kim, T. Song, and S. Pack, "An energy efficient message dissemination scheme in platoon-based driving systems," Energies, vol. 13, no. 15, pp. 1-23, 2020, doi: 10.3390/en13153940.

[29] T. Afrin and N. Yodo, "A survey of road traffic congestion measures towards a sustainable and resilient transportation system," Sustain., vol. 12, no. 11, pp. 1-23, 2020, doi: 10.3390/su12114660.

[30] S. Son and K. J. Park, "BEAT: Beacon inter-reception time ensured adaptive transmission for vehicle-to-vehicle safety communication," Sensors (Switzerland), vol. 19, no. 14, 2019, doi: 10.3390/s19143061.

[31] D. Punia and R. Kumar, "Experimental Characterization of Routing Protocols in Urban Vehicular Communication," Transp. Telecommun., vol. 20, no. 3, pp. 229-241, 2019, doi: 10.2478/ttj-2019-0019.

[32] K. Gao, F. Han, P. Dong, N. Xiong, and R. Du, "Connected vehicle as a mobile sensor for real time queue length at signalized intersections," Sensors (Switzerland), vol. 19, no. 9, pp. 1-22, 2019, doi: 10.3390/s19092059.

[33] Y. Chen, C. Lu, and W. Chu, "A Cooperative Driving Strategy Based on Velocity Prediction for Connected Vehicles with Robust Path-Following Control," IEEE Internet Things J., vol. 7, no. 5, pp. 3822-3832, 2020, doi: 10.1109/JIOT.2020.2969209.

[34] D. F. Xie, Y. Q. Wen, X. M. Zhao, X. G. Li, and Z. He, "Cooperative driving strategies of connected vehicles for stabilizing traffic flow," Transp. B, vol. 8, no. 1, pp. 166-181, 2020, doi: 10.1080/21680566.2020.1728590.

[35] T. Li, D. Ngoduy, F. Hui, and X. Zhao, "A car-following model to assess the impact of $\mathrm{V} 2 \mathrm{~V}$ messages on traffic dynamics," Transp. B, vol. 8, no. 1, pp. 150-165, 2020, doi: 10.1080/21680566.2020.1728591.

[36] L. Gao, Y. Hou, X. Tao, and M. Zhu, "Energy-Efficient Power Control and Resource Allocation for V2V Communication," IEEE Wirel. Commun. Netw. Conf. WCNC, vol. 2020-May, no. 2, pp. 1-2, 2020, doi: 10.1109/WCNC45663.2020.9120612. 